\title{
Gestión del conocimiento y productividad de una empresa constructora del Perú
}

\section{Knowledge and productivity management of a construction company in Peru}

DOI: $10.46932 / \mathrm{sfjdv2n4-017}$

Received in: March 1st, 2021

Accepted in: May 30th, 2021

\author{
Rubén Moisés Mauricio Avalos \\ Filiación Universidad César Vallejo \\ Correo:mauriciodata@gmail.com \\ Denisse Vanessa García Chacchi \\ Filiación Universidad César Vallejo \\ Correo: denissevanessagch@gmail.com \\ Nancy Lucía Merma Yépez \\ Filiación: Universidad César Vallejo \\ Correo: nancymerma2015@gmail.com \\ Ericka Janet Villamares Hernández \\ Filiación: Universidad Privada San Juan Bautista \\ Correo: ericka.villamares@upsjb.edu.pe
}

\begin{abstract}
RESUMEN
El estudio de investigación tuvo como objetivo determinar la relación entre la gestión del conocimiento en la productividad de la empresa CONSTRUCTORA PBG E.I.R.L. San Martín de Porres, 2020. El tipo de investigación fue de tipo básica, diseño correlacional, transversal. La muestra estuvo conformada por 70 colaboradores, con una población censal de 70 trabajadores, con instrumentos validados por expertos y una alta fiabilidad. La escala de percepción de la gestión del conocimiento, para el presente estudio presentó confiabilidad con Alfa de Cronbach de 0.788 indicando una fuerte consistencia interna, y la Escala de productividad, para la investigación presentó validez de confiabilidad con Alfa de Cronbach de 0.891 indicando una fuerte consistencia interna. Los resultados descriptivos de la variable gestión del conocimiento según los trabajadores el $35.7 \%$ lo considera deficiente, el $42.9 \%$ los considera regular y el $21.4 \%$ es considerado como eficiente; en la dimensión Creación del conocimiento, el $28.6 \%$ lo considera deficiente, el $50.0 \%$ los considera regular y el $21.4 \%$ es considerado como eficiente; en la dimensión Transferencia del conocimiento el $25.7 \%$ lo considera deficiente, el $45.7 \%$ los considera regular y el $28.6 \%$ es considerado como eficiente; en la dimensión Aplicación del conocimiento el 28.6\% lo considera deficiente, el $42.9 \%$ los considera regular y el $28.6 \%$ es considerado como eficiente. Los resultados nos indicaron la gestión del conocimiento se relaciona con la productividad se relacionan moderadamente con un (Rho 0,622 y p-valor 0,000)
\end{abstract}

Palabras clave: Gestión, conocimiento, productividad, constructora, trabajadores.

\begin{abstract}
The research study aimed to determine the relationship between knowledge management on the productivity of the company CONSTRUCTORA PBG E.I.R.L. San Martín de Porres, 2020. The type of research was basic, correlational, cross-sectional design. The sample consisted of 70 collaborators, with a census population of 70 workers, with instruments validated by experts and high reliability. The
\end{abstract}


knowledge management perception scale for the present study presented reliability with Cronbach's Alpha of 0.788 indicating strong internal consistency, and the Productivity Scale, for the investigation, presented reliability validity with Cronbach's Alpha of 0.891 indicating strong internal consistency. The descriptive results of the knowledge management variable according to the workers, $35.7 \%$ consider it deficient, $42.9 \%$ consider it regular and $21.4 \%$ consider it efficient; in the Knowledge creation dimension, $28.6 \%$ consider it deficient, $50.0 \%$ consider it regular and $21.4 \%$ consider it efficient; In the knowledge transfer dimension, $25.7 \%$ consider it deficient, $45.7 \%$ consider it regular and $28.6 \%$ consider it efficient; In the Knowledge Application dimension, 28.6\% consider it deficient, 42.9\% consider it regular and $28.6 \%$ consider it efficient. The results indicated that knowledge management is related to productivity and is moderately related to a (Rho 0.622 and p-value 0.000 )

Keywords: Management, knowledge, productivity, construction, workers.

\section{INTRODUCCIÓN}

A nivel mundial el sector empresarial de la construcción ocupa un sitial relevante en la economía de la gran mayoría los países dado que los beneficios son óptimos para el desarrollo empresarial. La gestión del conocimiento está tomando vital importancia en especial en las empresas en Estados Unidos, que están migrando a una economía que se fundamenta en el conocimiento, cambiando de este modo la forma de gestionar las empresas en especial los denominados bienes intangibles que vienen a ser el capital intelectual, lo que buscan las empresas que están gestionando el conocimiento es centrarse en la innovación y añadir valor lo más pronto en torno al conocimiento; estos cambios se están generando a partir de la búsqueda de mejores estrategias para incrementar la productividad. Al respecto la Universidad de Harvard en setiembre del 2018 publico un estudio acerca de que sectores empresariales no han adoptado la tecnología en sus procesos, el estudio revela que el sector construcción ocupa el segundo lugar como el sector que menos ha adoptado tecnología en sus procesos y muestra que son muy pocas las empresas que han evolucionado y adaptado al cambio.

Sin embargo, es importante señalar que en Perú las empresas muestran muchos puntos débiles en gestión del conocimiento, bajo la dirección de sus dueños (pequeñas empresas) en base a conocimientos producto de sus experiencias laborales y a que no utilizan sistemas de gestión puesto que la mayoría depende del personal que contratan por temporada. Muy por el contrario, las empresas empoderadas en nuestro país con inversionistas extranjeros se preocupan por las necesidades del cliente, acomodarse a las nuevas actualizaciones y sacar ventaja de las oportunidades que el mercado laboral con miras a la competitividad.

En el caso local, existe una incipiente gestión del conocimiento en la empresa CONSTRUCTOTA PBG, debido a la poca participación y toma de decisiones de los trabajadores debido a que existe poca motivación para compartir los conocimientos, así mismo no hay un buen uso de las tecnologías en cuanto 
al almacenamiento de información, (Ríos,2012). La empresa CONSTRUCTORA PBG es más en cuanto a sus trabajadores presentan desconocimiento de una adecuada gestión del conocimiento, que les permita realizar el cambio de conocimiento tácito a el conocimiento explicito, para que pueda ser estructurado, almacenado y compartido; hay mucha rotación de personal, contrato por temporada lo que ocasiona un bajo nivel de gestión de conocimiento y por ende poco compromiso por parte de los trabajadores lo que impide elevar el nivel de productividad. La baja productividad en la empresa en las áreas de ingeniería y proyectos ha motivado la realización del presente trabajo de investigación, teniendo por objetivo la de encontrar relación alguna entre la gestión del conocimiento con la productividad.

Los trabajos previos internacionales que podemos referir son: García (2019) en su tesis de maestría: Gestión del conocimiento y eficiencia en proyectos. caso empresa Robuspackel, su objetivo fue someter en prueba un estándar para la gestión adecuada del conocimiento para lo cual utilizo una práctica ya estandarizada de gestión de proyectos que permita medir el efecto que acarrea en la eficiencia que tienen los proyectos dentro de Robuspack. Para lo cual utilizo un enfoque cuantitativo, de carácter descriptivo, aplicó cuestionarios a 85 trabajadores para la elección de un modelo. Concluyó que se deben implementar modelos de acuerdo con las necesidades de la empresa y con ello se lograra elevar el nivel competitivo.

Gómez (2017) en su tesis doctoral: Convergencia de la gestión del conocimiento y el eLearning en el Portfolio Profesional, su objetivo fue analizar cuál es la percepción por parte de los futuros usuarios de este tipo de sistema de formación. Es una investigación descriptiva, Los resultados a los que llego el investigador es que el 85,71\% de los trabajadores quiere recibir formación en la empresa para mejorar su desempeño. Se concluyó que la convergencia entre el eLearning y la forma como se maneja el conocimiento es posible, estos sistemas de eLearning deberían incorporar funcionalidades de búsqueda independientes y que una adecuada gestión del conocimiento que adopte soluciones híbridas de personas y tecnología.

Trabajos previos nacionales como: Vélez (2018) en su tesis de maestría: Gestión del conocimiento y productividad laboral según los funcionarios de la Dirección Regional de Salud de Ica. El investigador se fijó por objetivo principal la de encontrar cual podría ser la relación que existe entre la forma de gestionar el conocimiento en relación con la productividad, para lograr ello uso el enfoque cuantitativo de naturaleza descriptiva-correlacional, de una población de 210 funcionarios obtuvo una muestra probabilística de 136 funcionarios a quienes se le aplicaron cuestionarios cuyos resultados fueron que existe una relación baja de 0,322 , con estos datos concluyó que se debe gestionar conocimientos para mejorar su productividad. 
Chauca (2018) en su tesis de maestría: Gestión del conocimiento y desempeño de empresas constructoras. La investigación tuvo por objetivo principal la de encontrar cuál podría ser la vinculación entre el sistema encargado de la gestión del conocimiento en relación con el desempeño de la empresa constructora mediante los indicadores de gestión, productividad, eficiencia y eficacia de las empresas. Para lo cual utilizó una metodología correlacional, se aplicó encuestas a los trabajadores, dando como resultado que el sistema de gestión se relaciona con el desempeño con Rho Spearman de ,733 relación alta, debido a la relación entre el nivel de producción y el logro de metas.

Es importante precisar definiciones de conocimiento, al respecto es considerada como un conjunto de creencias relacionadas con los fenómenos y las condiciones que lo afectan (Buck,2000), es también un acto de construcción de ideas según el punto de vista humano (Von Krogh, 1998), es señalada como una capacidad de resolución de una problemática (Liu, Chen \& Tsai,2005), otra definición seria el almacenamiento de información que toda persona tiene en su mente, cultura (Díaz \& Contreras, 2009).

En cuanto a la gestión del conocimiento, existen varias definiciones, es un método que permite transferir conocimientos de una persona a otra (Rodríguez,2011), desde el punto de vista empresarial consiste en el desarrollo de capacidades y actividades que permitirán diseñar nuevos conocimientos (Díaz,2007), otra concepción es tomada como un mecanismo que genera conocimientos dentro de una organización (Almashari, Zairi, Alathari,2002), así también refirieron como la generación de conocimiento que permite un aprendizaje organizacional (Oliveira y Caldeira,2008),consiste también en la creación de nuevos conocimientos a través de la interrelación con otras personas (Kyeonghee, 2012).

Presentó las siguientes dimensiones: Como primera dimensión, creación del conocimiento, se inicia y desarrolla con el individuo consiste en el hacer conocimiento no de forma rígida sino dinámica. Dimensión 2: Transferencia del conocimiento, consiste en la trasmisión de conocimientos a través de acciones encaminadas a compartir con otros y todos los procesos que se requieran para su transmisión. Dimensión 3: Aplicación del conocimiento, consiste en la utilidad práctica del conocimiento, así como también su productividad en beneficio de la organización (Nonaka y Takeuchi,2009). La importancia de generar conocimiento y gestionarlo está en saber utilizarlo adecuadamente y en beneficio de la institución. (Nonaka y Takeuchi, 2009) por ello es importante creas, transferir y aplicar conocimientos para obtener mejoras. Es importante porque toda adecuada gestión del conocimiento permitirá la innovación y el éxito empresarial (Oltra,2011), otro factor importante es que permite conocer conocimientos internos y que se desarrolle una cultura de aprendizaje (Pérez, Valle y Wiklund, 2007)

La productividad puede concebirse de diferentes maneras a nivel empresarial, así tenemos que es considerada como un nivel de vida de las empresas a lo largo del tiempo (Jaimes y Rojas, 2015), otra definición consiste en un conjunto de acciones y productos que tienen que ver con la formación, 
experiencia y el entrenamiento de los trabajadores dentro de una empresa(Tovar,2017), consiste también en las habilidades y destrezas a través de los conocimientos adquiridos para hacer de la empresa más eficiente y productiva(Reina,2016), consiste en incrementar la eficacia y eficiencia a través del trabajo de los trabajadores que generen satisfacción en los clientes (Aguirre y Pelekais,2014), es importante resaltar que la productividad es la relación entre la actividad y todos aquellos recursos que se han empleado con la finalidad de alcanzar las metas trazados (Chiavenato,2007), es el cúmulo de esfuerzos para producir (Singh, 2008).

Los criterios de productividad son: a) Eficiencia, son los recursos empleados y los logros alcanzados por la empresa, b) Efectividad, es la vinculación que hay entre los resultados que se obtienen y aquellos que se esperan, c) Eficacia, consiste en la medición de los objetivos establecidos. Existen factores para mejorar la productividad como: a) Factores internos, que pueden ser duros referidos a los productos, tecnología y materias primas y los duros referidos a la fuerza y métodos de trabajo, b) Factores externos, referida a los ingresos, inflación y competitividad dentro de una organización (Bain, 2003). Las ventajas que permite la productividad son: a) Determina si los recursos estarán disponibles, b) Toma decisiones que generen beneficios. c) Reduce costos y procesos innecesarios. d) Mide los indicadores económicos de la empresa. e) Mide los procesos de producción. f) Persigue metas específicas. g) Realiza análisis de sus riesgos y oportunidades (Mallar,2013).

Presenta tres dimensiones: Dimensión 1: Eficiencia, consiste en el cumplimiento de funciones específicas. Dimensión 2: Eficacia, se refiere a la ejecución de una actividad sin tomar en cuenta el empleo de medios que se tienen. Dimensión 3: Competitividad, "consiste en el rendimiento y esfuerzos que realizan los trabajadores para competir en un mercado comercial (Rivas, 2016). Esto reviste vital importancia pues establece una brecha que significa una superioridad competitiva para la empresa en el sector donde se desarrolla. Analizando el tema de la productividad dentro de un entorno laboral se reduce en comparación frente a otras empresas del mismo sector, esto puede llegar a generar una desventaja competitiva. A si mismo si los costos en la producción suben, estas empresas pierden ventas competitivas ya que los clientes elegirán proveedores a un costo menor. Por ello reviste vital importancia entender que al mejorar la productividad no únicamente nos centremos en hacer las cosas óptimamente, sino que también se debe de hacer las cosas correctas. (Prokopenko, 1999)

\section{MÉTODO}

Se empleó el enfoque cuantitativo, porque su intención es recabar datos y procesarlos a través de la estadística, el método hipotético - deductivo porque se basa en la aprobación de hipótesis para poder sacar conclusiones sobre el fenómeno de estudio (Ramos, 2015). La población estuvo constituida por La 
población fue de 70 trabajadores y muestra fue censal conformada por 70 trabajadores de la empresa CONSTRUCTORA PBG E.I.R.L. San Martín de Porres; se utilizó la encuesta. Las técnicas e instrumentos de recolección de datos fueron: Cuestionario para la Gestión del conocimiento Nonaka y Takenchi (2009) y el cuestionario para la productividad. Para realizar el análisis de los datos, se utilizó la prueba de normalidad o de bondad de ajuste de Kolmogorov de Srmirnov, posteriormente, se empleó la estadística descriptiva y la inferencial, se procesó la información mediante software estadístico SPSS 23 y para la correlación de los resultados obtuvo con el estadígrafo no paramétrico el Rho Spearman.

\section{RESULTADOS}

Sobre los resultados cuantitativos tenemos:

Tabla 1. Relación de las variables y sus dimensiones.

\begin{tabular}{|c|c|c|c|c|c|}
\hline Hipótesis & $\begin{array}{l}\text { Variables * } \\
\text { Correlación }\end{array}$ & $\begin{array}{c}\text { Rho- } \\
\text { Spearman }\end{array}$ & $\begin{array}{c}\text { Significatividad- } \\
\text { Bilateral }\end{array}$ & $\mathrm{N}$ & Nivel \\
\hline $\begin{array}{l}\text { Hipótesis } \\
\text { general }\end{array}$ & $\begin{array}{c}\text { Gestión del } \\
\text { conocimiento* } \\
\text { Productividad }\end{array}$ &, $622^{* *}$ & ,000 & 70 & $\begin{array}{c}\text { Considerab } \\
\text { le }\end{array}$ \\
\hline $\begin{array}{c}\text { Hipótesis } \\
\text { específica- } \\
1\end{array}$ & $\begin{array}{c}\text { Gestión del } \\
\text { conocimiento* } \\
\text { Eficiencia }\end{array}$ &, $428^{*}$ & ,000 & 70 & Moderado \\
\hline $\begin{array}{c}\text { Hipótesis } \\
\text { específica- } \\
2\end{array}$ & $\begin{array}{c}\text { Gestión del } \\
\text { conocimiento* } \\
\text { Eficacia }\end{array}$ &, $484^{* *}$ & ,000 & 70 & Moderado \\
\hline $\begin{array}{c}\text { Hipótesis } \\
\text { específica- } \\
3\end{array}$ & $\begin{array}{c}\text { Gestión del } \\
\text { conocimiento* } \\
\text { Competitividad }\end{array}$ &, $545^{* *}$ & ,000 & 70 & $\begin{array}{c}\text { Considerab } \\
\text { le }\end{array}$ \\
\hline
\end{tabular}

Como resultado final se logró probar las hipótesis para ello se establecieron las hipótesis nulas y las hipótesis alternas, se rechazaron las hipótesis nulas y se aceptaron las alternas de las variables y de las dimensiones - variable, se empleó el Rho de Spearman que indicó para la hipótesis general (Gestión del conocimiento y productividad) el nivel de correlación dando como moderado (Rho 0,622 y p-valor 0,000); en el caso de la hipótesis especifica-1 (Creación del conocimiento* productividad) el nivel de correlación hallado es moderado (Rho 0,428 y p-valor 0,000); para el caso de la hipótesis especifica-2 (Transferencia del conocimiento* productividad) el nivel encontrado de correlación es moderado (Rho 0,484 y p-valor 0,000); y finalmente para la hipótesis especifica-3 ( Aplicación del conocimiento* 
productividad) el nivel de correlación hallado es moderado con (Rho 0,545 y p-valor 0,000), para la totalidad de los casos en el nivel 0,01.

\section{DISCUSIÓN}

Para el caso de la hipótesis general. La gestión del conocimiento se relaciona con la productividad nos indicó que el nivel de correlación encontrado es moderado (Rho 0,622 y p-valor 0,000); estos resultados difieren con los de García (2019) al señalar que para que pueda darse una gestión optima del conocimiento se requiere de una adecuada implementación de un modelo de gestión que evalúe el impacto de este en la eficiencia de los proyectos, sin embargo, para Gómez (2017) señaló que se requiere contar con sistemas digitales para obtener una gestión eficaz del conocimiento, estos resultados fueron distintos a los nuestros que existe un nivel moderado de gestión del conocimiento porque si bien es cierto se crea y transfiere el conocimiento de cada área de construcción específica pero no se aplican muchas veces los conocimientos esto se evidencia con el resultado obtenido en el nivel regular de productividad de la empresa constructora.

Asimismo, se comprueba que entre gestión del conocimiento y las tres dimensiones de la productividad (Eficiencia, Eficacia y Competitividad), existe una relación directa y considerable de $54.9 \%, 68.2 \%, 68.7 \%$ y $55.3 \%$ respectivamente, demostrando que efectivamente la gestión del conocimiento si mejoran significativamente la productividad en las tres dimensiones analizados, reforzando con lo manifestado por Torres y Lamenta (2015) quienes señalaron que las empresas se encuentran en procesos cambiantes, propios del mundo globalizado, es por ello la que se requiere la obtención de nuevos conocimientos y habilidades que permitan innovar para lograr posiciones competitivas con el apoyo de los sistemas de información al contrario, Lacu (2017) señaló la importancia de hacer uso del conocimiento, con la finalidad de lograr que una empresa sea mucho más inteligente y a su vez mucho más competitiva. A su vez estos resultados son diferentes con los hallados, puesto que los conocimientos adquiridos y de las experiencias de los trabajadores es el que prevalece en la empresa, incidiendo muchas veces en el obsoletísimo, rutina y no ha permitido la apertura de nuevos conocimientos que les permite el aumento sustancial de la productividad en la empresa constructora.

En cuanto a la hipótesis especifica-2 La transferencia del conocimiento se relaciona con la productividad indicó que el nivel de correlación hallado es moderado con un (Rho 0,484 y p-valor 0,000); estos resultados difieren con Vélez (2018) que enfatizó en la necesidad de propiciar los espacios de interacción que logren la apertura a la creación y transferencia del conocimiento por intermedio de las plataformas de uso gratuitas y así mismo motivar al personal para lograr incrementar su productividad 
laboral, al contrario, Chauca (2018) señaló que existe una alta correlación ,733 la transferencia de conocimientos guarda relación con la productividad de las empresas constructoras.

Finalmente para el caso de la hipótesis especifica-3 La aplicación del conocimiento se relaciona con la productividad indicó que el nivel encontrado de correlación es moderado con un (Rho 0,545 y pvalor 0,000), al respecto, Vega (2017) señaló una alta relación y que se debe gestionar eficazmente el conocimiento de los trabajadores según la labor que realizan, así mismo el desarrollo de capacidades competitivas que impliquen crecimiento de las empresas, al contrario, Borda (2015) señaló que existe una baja correlación debido a que no se aplica correctamente el conocimiento entre los trabajadores y se pone en evidencia en el poco incremento en cuanto a la productividad se refiere de la empresa constructora.

\section{CONCLUSIONES}

Como primera conclusión tenemos que, la gestión del conocimiento tiene una relación moderada con la productividad (Rho 0,622 y p-valor 0,000), catalogándose como correlación positiva y directa; con esto se logró el objetivo general de la investigación, que era la de determinar la relación entre la gestión del conocimiento en la productividad de la empresa CONSTRUCTORA PBG E.I.R.L., a mayor gestión del conocimiento se tendrá mayor productividad.

Como segunda conclusión tenemos que, la creación del conocimiento tiene una relación moderada con la productividad (Rho 0,428 y p-valor 0,000 ), catalogándose como correlación positiva y directa, con la cual se logró el primer objetivo específico de la investigación, que era la de determinar la relación entre la creación del conocimiento en la productividad de la empresa CONSTRUCTORA PBG E.I.R.L., a una mayor creación del conocimiento se tendrá mayor productividad.

Como tercera conclusión tenemos que, en lo que se refiere a la transferencia del conocimiento hay una relación moderada con la productividad (Rho 0,484 y p-valor 0,000), catalogándose como correlación positiva y directa. Con esto se logró el segundo objetivo específico de la investigación, que era la de determinar la relación entre la transferencia del conocimiento en la productividad de la empresa CONSTRUCTORA PBG E.I.R.L., con el incremento de la transferencia del conocimiento se tendrá mayor productividad.

Finalmente, como cuarta conclusión tenemos que, la aplicación del conocimiento se relaciona moderadamente con la productividad (Rho 0,545 y p-valor 0,000), catalogándose como correlación positiva y directa. Finalmente, con esto se logró el último objetivo específico de la investigación, que era la de determinar la relación entre la aplicación del conocimiento en la productividad de la empresa 
CONSTRUCTORA PBG E.I.R.L., con el incremento de la aplicación del conocimiento se tendrá mayor productividad. 


\section{REFERENCIAS}

Aguirre, R. y Pelekais, C. (2014). El liderazgo de servicio como instrumento fundamental de productividad en las organizaciones universitarias de gestión privada. Telos, 16() 315-326. Recuperado de http://www.redalyc.org/articulo.oa?id=99331125008

Almashari, M., Zairi, M., y Alathari, A. (2002). An empirical study of the impact of knowledge management on organizacional performance. J. Comp. Inf. Syst. 42: 74-82.

Bain. D. (2003). La productividad. Colombia: McGraw-Hill.

Borda, J. (2015) Productividad total de los factores del sector industrial en el departamento de Antioquia para el periodo 20002010 Retrieved from https://ciencia.lasalle.edu.co/economia/284

Bück, J. (2000) Gestión del conocimiento, Asociación Española de Normalización y Certificación, AENOR, España.

Chauca, A. (2018) Gestión del conocimiento y desempeño de empresas Constructoras (Tesis de maestría) Universidad Nacional Federico Villarreal.

Chiavenato, I. (2007). Introducción a la teoría general de la administración ( $8^{\text {a }}$ edición ed.). México: Mc Graw Hill.

Díaz, L. (2007) Gestión del conocimiento y del capital intelectual: una forma de migrar hacia empresas innovadoras, productivas y competitivas. Revista Escuela de Administración de Negocios, núm. 61, septiembre-diciembre, 2007, pp. 39-67 Universidad EAN Bogotá, Colombia. Recuperado:https://www.redalyc.org/pdf/206/20611495005.pdf

Díaz, M. (2009) El factor humano como elemento dinamizador del proceso empresarial en la gestión de la información y conocimiento. ACIMED v.20 n.5 Ciudad de La Habana nov. 2009. Recuperado: $\quad$ http://scielo.sld.cu/scielo.php?script=sci_arttext\&pid=S1024$\underline{94352009001100004}$

García, J. (2019) Gestión del conocimiento y eficiencia en proyectos. caso empresa Robuspackel. CIATEQ, México. Recuperado: https://www.ciateq.mx/index.php/conoce-ciateq.html

García, R. (2015) Gestión del conocimiento y eficacia de las organizaciones. Santiago, Chile (Tesis de maestría) Universidad de Valparaíso, Chile.

Gómez, M. (2017) Convergencia de la Gestión del Conocimiento y el eLearning en el Porfolio Profesional (Tesis de maestría) Universidad Autónoma de Madrid, España.

Jaimes, L. y Rojas, M. (2015). Una mirada a la productivity laboral para las pymes de Confecciones. Iteckne, $12 \quad$ (2), 177-187. Recuperado: http://www.scielo.org.co/scielo.php?script=sci_arttext\&pid=S1692-

$17982015000200009 \& \operatorname{lng}=\mathrm{en} \& \operatorname{tn} \mathrm{ln}=\mathrm{es}$

Kyeonghee, J. (2012). An Understanding of Optimal Knowledge Management for Social Work Practice: Based on a Process-Oriented Conceptualisation of Knowledge Integration. 
Lacu, M. (2017) El incremento de la productividad organizacional a través del uso de la gestión del conocimiento. (Tesis de maestría) Universidad Pontificia Comillas de Madrid, España. Recuperado: https://dialnet.unirioja.es/servlet/tesis?codigo $=125043$

Liu, P., Chen, W. y Tsai, Ch. (2005). An empirical study on the correlation between the knowledge management method and new product development strategy on product performance in Taiwan's industries. Technovation25: 637-644.

Mallar, M, (2010) La gestión por procesos: un enfoque de gestión eficiente Revista Científica "Visión de Futuro", vol. 13, núm. 1, enero-junio, 2010 Universidad Nacional de Misiones Misiones, Argentina

Nonaka, I. y Takeuchi, H. (2009). Cómo las compañías japonesas crean la dinámica de la innovación. México: Oxford University Press.

Nuñez P. (2008): Aproximación metodológica para introducir la gestión del aprendizaje en las organizaciones y comunidades, Seminario Iberoamericano sobre Tendencias Modernas en Gerencia de la Ciencia y la Innovación Tecnológica. IBERGECYT'2002, Editorial Academia, pp. 136 - 148, La Habana.

Oliveira, M. y Caldeira, M. (2008). Um «framework» para a gestão do conhecimento nas organizações. Rev. Portuguesa e Brasileira de Gestão, Lisboa.

Oltra, V. (2011). La Complejidad del Conocimiento: Retos para su eficaz creación y transferencia en la organización innovadora. Estudios Gerenciales - Universidad Icesi.

Pérez, A., Valle, R., \& Wiklund, J. (2007). Innovation and imitation as Sources of sustainable competitive advantage. Management Research,5(2), 67 - 79, 2007

Prokopenko, J. (1999). La gestión de la productividad. C.V. México: Editorial LIMUSA S.A.

Ramos, J. (2015) Métodos, técnicas y procedimientos de investigación. 2da Edición, Editorial jurídica Grijley, Lima, Perú.

Reina, R; (2016). Productividad de recursos humanos, innovación de producto y desempeño exportador: Intangible Capital, vol. 12, núm. 2, 2016, pp. 619-641 Universitat Politécnica de Catalunya Terrassa, España. Recuperado: http://www.redalyc.org/articulo.oa?id=54944740010

Ríos, T. (2012) La gestión del conocimiento y la educación superior universitaria. Gestión en el Tercer Milenio, Rev. de Investigación de la Fac. de Ciencias Administrativas, UNMSM (Vol. 15, N 30, Lima, Diciembre 2012). Disponible en file://C:/Users/USUARIO/Downloads/8797-30525-1-PB.pdf

Rivas, L. (2016). Dirección estratégica. Proceso de la organización en el siglo XXI. México: Trillas. Rodríguez, A. (2011). Gestión del conocimiento "On-Line" http://www.slideshare.net/arlinesrodriguez/gestion-del-conocimiento-20-072010

Singh, H. (2008). Human factor and organizational productivity in textile industry and agricultural machinery industry in north India. Thesis summary Submitted to the Faculty of Business Management \& Commerce. For the degree of Doctor of Philosophy. Panjab University, Chandigarh. India. 
Torres, K y Lamenta, P. (2015) La gestión del conocimiento y los sistemas de información en las organizaciones. Revista Científica Electrónica de Ciencias Gerenciales Vol./ núm. 32 (año 11) pág. 3-20 / Scientific e-journal of Management Sciences.Recuperado: http://www.redalyc.org/html/782/78246590001/

Tovar, B. (2017). La teoría del capital humano-llevada a la práctica en las ciudades de aprendizaje. Nóesis. Revista de Ciencias Sociales y Humanidades,26 (51), 45-56. Recuperado: https://dialnet.unirioja.es/servlet/articulo?codigo $=5480847$

Vega, S. (2017) La gestión del conocimiento y su relación con el crecimiento empresarial de las MIPYMES (Tesis de maestría) Universidad César Vallejo.

Vélez, R. (2018) Gestión del conocimiento y productividad laboral según los funcionarios de la Dirección Regional de Salud de Ica. (Tesis de maestría) Universidad César Vallejo.

Von, K. (1998). Care in knowledge creation, California Management Review, vol. 40, nº 3, pp. 133-153. 